\title{
Polyatomic trilobite Rydberg molecules in a dense random gas
}

\author{
Perttu J. J. Luukkd* and Jan-Michael Rost \\ Max Planck Institute for the Physics of Complex Systems, Dresden, Germany
}

(Dated: November 22, 2017)

\begin{abstract}
Trilobites are exotic giant dimers with enormous dipole moments. They consist of a Rydberg atom and a distant ground-state atom bound together by short-range electron-neutral attraction. We show that highly polar, polyatomic trilobite states unexpectedly persist and thrive in a dense ultracold gas of randomly positioned atoms. This is caused by perturbation-induced quantum scarring and the localization of electron density on randomly occurring atom clusters. At certain densities these states also mix with a s-state, overcoming selection rules that hinder the photoassociation of ordinary trilobites.
\end{abstract}

If an atom is excited to a Rydberg state $n$ in a sufficiently dense gas, the range of its electron cloud $\left(\propto n^{2}\right)$ can be large enough to reach a nearby ground-state atom. For some elements the short-range electron-atom polarization interaction is attractive, and in very low temperatures it can create a bound state - an exotic giant molecule thousands of atomic units in size [1].

Rydberg molecules separate into two classes due to quantum defects affecting low angular momentum states of the Rydberg electron. Low- $l$ molecules are created from energetically separated states with $l \lesssim 2$ (typically an s-state). These molecules have relatively uniform electron densities and shallow BornOppenheimer (BO) potentials. In contrast, quasidegenerate high- $l$ states combine into much more deeply bound molecules where the Rydberg electron localizes on the distant ground-state atom. As a result, these so-called trilobite molecules have permanent dipole moments in the kilodebye range, making them very sensitive to external fields and a promising candidate for realizing exotic, strongly correlated systems.

Both classes of Rydberg molecules have been observed experimentally [2-10]. Because selection rules inhibit the photoassociation of pure high- $l$ trilobites, the production of highly polar Rydberg molecules requires circumstances where the two classes mix into a hybrid with a suitable mixture of low- and high- $l$ character. This has been only recently achieved for certain elements and Rydberg states using nearly integer quantum defects [9, 11] or resonant hyperfine splitting [10].

In sufficiently high densities the Rydberg electron can reach and bind several ground-state atoms. Polyatomic s-state Rydberg molecules with up to five constituent atoms have been observed in experiments [12, 13]. For an isolated s-state the effect of additional atoms to the electronic wave function can be neglected and very high densities can be studied using a mean-field model that adds up contributions for each atom from the dimer BO potential [14, 15]. This approach does not apply for the trilobite class, where the electron density depends on the positions of all perturbing atoms.

Polyatomic trilobites have been studied in constrained geometries where all atoms share a common distance $R$ to the Rydberg core [16, 17]. This allows solving the electronic problem efficiently in the basis of the dimer states and allows properties such as the BO potential to be studied as a function of the single coordinate $R$. However, such control on the atom positions is very difficult to achieve in experiments [17].
Here we study the high- $l$ trilobite states in a dense gas where the electron interacts with a large number of randomly positioned atoms. We show that highly polar, polyatomic trilobite states not only exist in high density, but counterintuitively, their dipole moment even increases with increasing density. We also show that a high density provides a novel and general way to mix s-state character into the trilobite states and thus make them accessible with conventional spectroscopy.

Our findings are explained by two key effects. Firstly, the recently discovered mechanism of perturbation-induced quantum scarring [18] explains why perturbations from randomly arranged atoms around the Rydberg excitation promote the formation of ordered eigenstates as a result of classical symmetries in the unperturbed system. Secondly, inevitable random fluctuations in the local density of the gas produce loose clusters that collectively attract electron density. Combined, these effects cause surprisingly polar "super-trilobite" states to commonly form in a sufficiently dense random gas.

Atomic units are used throughout unless otherwise noted.

Computational model. - To isolate the essential trilobite physics in a high density we use a model that minimally generalizes the original dimer trilobite calculations [1] to more constituent atoms. The Hamiltonian of the Rydberg-excited electron is $H=H_{0}+V$ where $H_{0}$ contains the Coulomb interaction with the Rydberg core and the quantum defects, and $V=\sum_{p} V_{\mathrm{s}}\left(\boldsymbol{R}_{p}\right)$ is the total perturbation from $N$ atoms fixed in space at locations $\left\{\boldsymbol{R}_{p}\right\}$. In this we assume that the polarizations of atoms are independent. Nearby atoms can, depending on the geometry, either increase or suppress each others' polarization, but for the densities studied here this should be a small effect compared to the direct polarization by the electron.

Because the range of the electron-atom interaction $V_{\mathrm{s}}$ is very short compared to the extent of the Rydberg electron, it can be modeled with the Fermi pseudopotential [19]

$$
\left\langle\Psi_{2}\left|V_{\mathrm{s}}(\boldsymbol{R})\right| \Psi_{1}\right\rangle:=2 \pi a_{\mathrm{s}} \Psi_{2}^{*}(\boldsymbol{R}) \Psi_{1}(\boldsymbol{R})
$$

where $a_{\mathrm{s}}$ is the s-wave scattering length of the perturbing atom. The Fermi model can be improved by adding terms corresponding to higher partial wave scattering [20]. The next term, corresponding to p-wave scattering, depends on the gradients of the wave-functions at position $\boldsymbol{R}$, and gives rise to three "butterfly states" in the dimer case [21]. However, it is the first term (1) 
that gives rise to the original trilobites. The p-wave term also formally diverges at the location of shape resonances.

To model the energy-dependence of $a_{\mathrm{s}}$ we use the modified effective range theory [22] expression

$$
a_{\mathrm{s}}(k)=a_{\mathrm{s}}(0)+\frac{\pi}{3} \alpha k,
$$

where $\alpha$ is the polarizability of the atom. The wave number $k$ is set by the semiclassical relation $E=k^{2} / 2-R^{-1}$, where $R$ is the distance to the Rydberg core. As the atomic species we use ${ }^{87} \mathrm{Rb}$ with $a_{\mathrm{s}}(0)=-16.1$ (spin triplet scattering) and $\alpha=319.2$ [23]. Quantitatively more accurate expressions for $a_{\text {s }}$ exist [24], but Eq. (2) provides a simple analytical formula that contains the key qualitative behavior: atoms near the classical turning point ( $k$ small $)$ are most attractive ( $a_{\mathrm{s}}$ most negative), and the strength of the attraction decreases at smaller $R$.

The total perturbation $V$ is not strong enough to mix states in different degenerate hydrogenic manifolds for the densities and principal quantum numbers considered here. For solving the eigenpairs of $H$ for a given $n$ we therefore use a basis of all hydrogen eigenstates whose energy, taking into account quantum defects, is $\left.E \in] E_{n-1}, E_{n}\right]$, where $E_{n}=-1 /\left(2 n^{2}\right)$. For the Rb quantum defects we use values tabulated in Ref. 24

The positions $\left\{\boldsymbol{R}_{p}\right\}$ of the perturbing atoms are sampled within the classically allowed radius $r_{\mathrm{c}}=2 n^{2}$ from a Poisson point process with a uniform density $\rho$. Some atoms will fall too close to the Rydberg core for Eq. (2) to be accurate, but due to the overall diminishing of $V_{\mathrm{s}}$ at small $R$ these atoms do not contribute significantly.

Trilobite states in high density. - The trilobite state is the lowest energy eigenstate of $H$ that forms out of the quasidegenerate high- $l$ states $(l \geq 3$ for $\mathrm{Rb}$ ). For $N=1$ this state localizes around the single ground-state atom and acquires a large dipole moment. Simple intuition suggests that, since the atoms are arranged randomly with a uniform density, a large number of additional atoms simply smears out the electron density into a featureless blob with zero dipole moment. Instead, solving the eigenstates reveals that they are often highly polar. At $n=50$ and $\rho=10^{16} \mathrm{~cm}^{-3}$, corresponding to over $10^{3}$ atoms in the classically allowed volume, $84 \%$ of trilobite states have a dipole moment greater than 1 kilodebye $(\mathrm{kD})$. Figure 1 shows an example of a trilobite state with dipole moment $|\boldsymbol{d}|=6 \mathrm{kD}$ (2360 a.u.) [26]. Far from a featureless blob, the state resembles the dimer trilobite, except that the cylindrical symmetry of the $N=1$ case is broken into approximately elliptic tracks of high probability density that orbit the Rydberg core.

To explain this oddly ordered structure it is beneficial to notice a connection to a phenomenon recently observed in a related physical setting. Impurities perturbing a two-dimensional quantum dot produce "scarred" high-energy eigenstates, i.e., eigenstates that concentrate around paths of classical periodic orbits (POs), even when the impurities are randomly placed [18]. This perturbation-induced (PI) scarring is similar in appearance to ordinary quantum scarring [27, 28], but a key difference is that in PI scarring the corresponding classical PO exists only in the unperturbed system and the scars exist only in

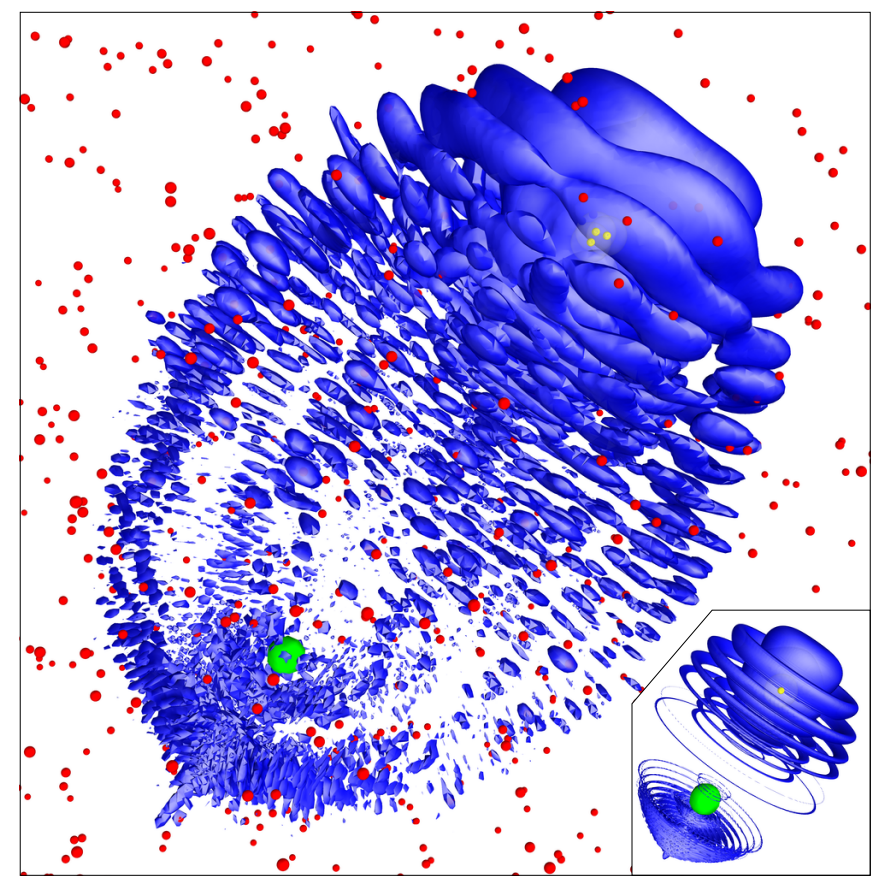

FIG. 1. Electron density of a highly polar $n=50$ rubidium trilobite state at atom density $\rho=10^{16} \mathrm{~cm}^{-3}$. The electron density is visualized using a contour surface (in blue) that contains $42 \%$ of the total probability mass. The small (red) spheres denote the locations of the perturbing atoms. For visibility, their radius is approximately twice the electron-atom scattering length. The electron density is localized around the highlighted cluster of three atoms (in yellow). The Rydberg core (in green) is at the origin, and the radius of the spherical cloud of atoms is the classically allowed radius $r_{\mathrm{c}}=5000$. For comparison, the inset shows an equal visualization of the ordinary trilobite state [1] with $n=30$ and a single atom at $R=1232$. Visualized with Mayavi [25].

the perturbed system - perturbing the system with impurities unmasks a hidden classical order.

The mechanism of PI scarring [18] requires two general ingredients, both of which are present in the current system: the unperturbed system needs to be separable, and the individual perturbations need to have a short spatial range.

If the unperturbed system is separable, applying the semiclassical Bohr-Sommerfeld quantization formula shows that at high energies its eigenstates are bunched into quasidegenerate sets. Each such "resonant set" corresponds to a family of classical POs, and this connection ensures that some linear combinations of the resonant states are scarred by corresponding POs.

A moderate perturbation produces eigenstates that are linear combinations of a single resonant set. By the variational theorem, the eigenstates corresponding to extremal eigenvalues extremize the Hamiltonian. Because the resonant states are nearly degenerate, this essentially extremizes the perturbation. In this extremization the scarred states have an advantage if the perturbations have a short range: scarred states can very effectively maximize (minimize) the perturbation by selecting paths that hit atypically many (few) perturbations. As a result, the extremal eigenstates arising from each resonant set often 
contain scars of the corresponding PO.

In the present system the separable unperturbed system is a hydrogen-like Rydberg atom, and the short-range electron-atom interactions create the localized perturbations. The resonant sets are the constant $n$ manifolds [29], the corresponding POs are Kepler orbits with a fixed energy, and the maximally scarred linear combinations are the stationary elliptic states [30].

The trilobite state essentially minimizes $V$ within the set of quasidegenerate high- $l$ states, i.e., it maximizes the electron density on the perturbing atoms. However, since it is confined within the quasidegenerate set, it has only limited capability to do so. The eigenstates scarred by Kepler orbits - guaranteed by semiclassics to exist - often represent the best available solution. Since a single Kepler orbit is confined to a plane, a single scar can only coincide with a small fraction of the perturbing atoms. Therefore it is unlikely that eigenstates localize perfectly on a single orbit, as in Ref. 18, but instead a scarred state likely contains scars of several orbits. The connection between Kepler orbits and the dimer trilobite state was also studied in Ref. 31

If several atoms perturb the system it is also important to consider their relative weights in the minimization of $V$. This weight is essentially given by the average value of $V_{\mathrm{s}}\left(\boldsymbol{R}_{p}\right)$, i.e., the dimer BO potential at interatomic distance $R_{p}$. Atoms at positions corresponding to a deep dimer potential give a larger contribution to the total $V$ and are thus favored, improving the localization.

A final and crucial point is that while the overall mean density of atoms is uniform, the individual random snapshots of $\left\{\boldsymbol{R}_{p}\right\}$ are not. Random fluctuations in the local density produce clusters where a few atoms happen to reside close to each other. At large $R$ the local wavelength of the electron is large, and even a relatively loose cluster of atoms can fit completely inside one half-wave. Such a cluster then acts as a single "superperturber" that can completely dominate in the minimization of $V$. As an example, the state in Fig. 11 is strongly localized around a cluster of three atoms located within 130 a.u. of each other. Because the available volume $\left(\alpha n^{6}\right)$ is large, the existence of such clusters has a surprisingly high probability. This is essentially a spatial analogue of the famous birthday paradox [32].

It is important to note that the clusters can be fairly sparse. At $n=50$ clusters with atomic separations between 100 and 300 a.u. seem to be common in high dipole moment trilobite states, and even larger clusters are possible for higher $n$.

The dominating nature of the clusters simplifies estimating the stability of the polar trilobite configuration, as it is sufficient to study only the few-body problem of the cluster. However, this is still substantially more difficult than in the dimer case. Adding a p-wave scattering term to $H$ also likely produces a separate class of "butterfly states", just as in the dimer case [21]. The states separate into s-wave dominated trilobites and p-wave dominated butterflies because the same state cannot simultaneously maximize both the wave function and its gradient at the position of the cluster.

The clusters complete the explanation for highly polar trilobite states at high density. There is a high probability that a cluster of atoms occurs somewhere in the large volume of
Average number of perturbers $\langle N\rangle$

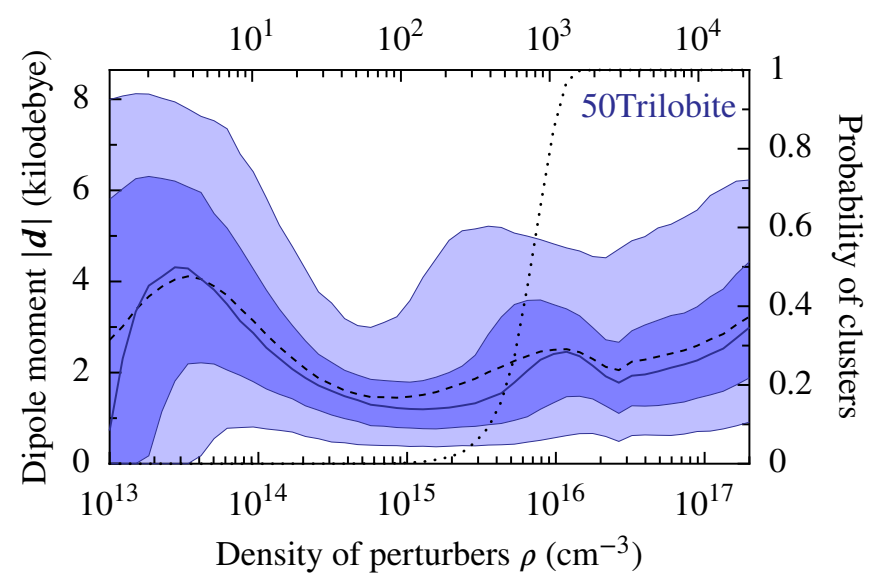

FIG. 2. Probability distribution of the dipole moment of the $n=50$ trilobite state as a function of the atom density $\rho$. The solid line shows the median value and the edges of the two shaded regions denote the 5th, 25th, 75th, and 95th percentiles. The dashed line denotes the mean value. The dotted line shows the probability that a snapshot of $\left\{\boldsymbol{R}_{p}\right\}$ contains at least one cluster where three atoms are located within 300 a.u. of each other. The values are estimated from an ensemble of $10^{4}$ snapshots of the atom locations.

space where the dimer potential is deep, and this cluster then dominates in the minimization of $V$. The states within the quasidegenerate high- $l$ manifold that most efficiently maximize the electron density on the cluster are scars of Kepler orbits whose apoapsis, where the electron density is higher, occurs near the cluster. Atoms not in the cluster have only a minor role in the formation of the state, but they do break the cylindrical symmetry and give the trilobite a skeletal appearance in which individual Kepler orbit PI scars can be resolved, as in Fig 1

The dipole moment.- A key property that makes the dimer trilobites interesting is their extraordinarily large dipole moment. An important consequence of the previously described mechanism is that the trilobite states can retain this property at high densities. Computing the dipole moment also provides a way to quantify how common the polar trilobite states are for a given density $\rho$.

Figure 2 shows the probability distribution of the dipole moment $|\boldsymbol{d}|$ as a function of $\rho$ for the $n=50$ trilobite state. As the average number of perturbing atoms $\langle N\rangle$ increases, the competition between atoms causes the dipole moment to initially decrease. However, as clusters of atoms become more likely the dipole moment starts to increase as a function of $\rho$. As non-intuitive as it is, adding atoms randomly and uniformly in space makes the trilobite states more polar. Other principal quantum numbers $n$ give similar results [26]. Fig 2 also shows how the probability of a sufficiently small cluster of three atoms grows as a function of $\rho$. The existence of a cluster somewhere in the classically allowed region is almost certain at $\rho=10^{16} \mathrm{~cm}^{-3}$, even though the probability of three atoms existing in a given, cluster-sized volume is still very small $\left(10^{16} \mathrm{~cm}^{-3}=1.5 \times 10^{-9}\right.$ a.u. $)$. 


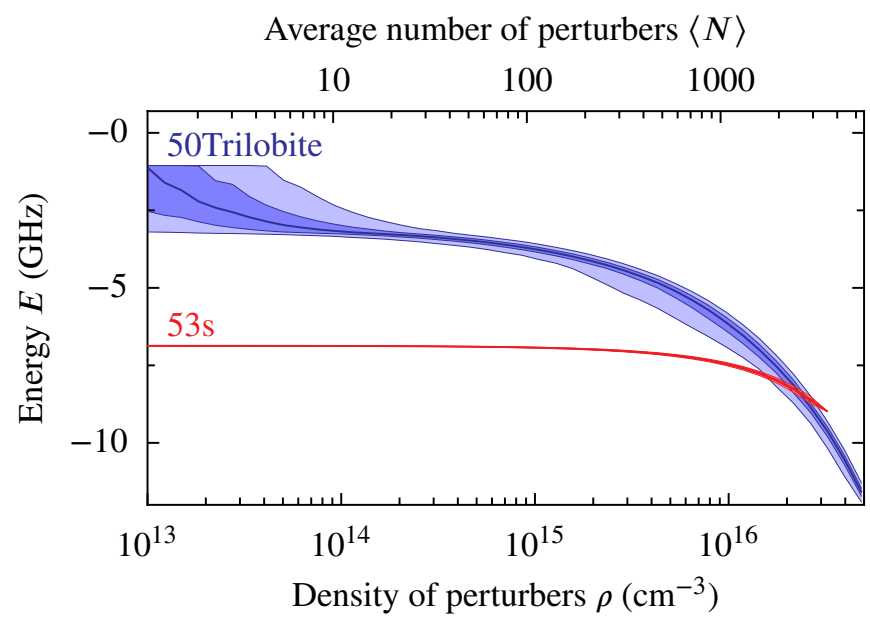

FIG. 3. Probability distribution of the energy of the $n=50$ trilobite state and the $53 s$ state as a function of the atom density $\rho$. The zero of energy is chosen at the degenerate $n=50$ hydrogen manifold. The probability distribution is visualized through its mean and percentile curves and estimated from an ensemble as in Fig. 2

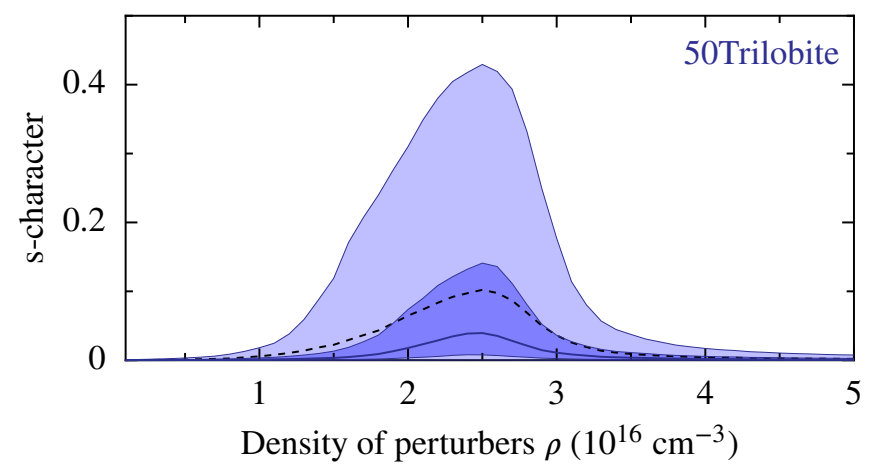

FIG. 4. Distribution of the magnitude of $l=0$ character in the $n=50$ trilobite state, i.e., the squared overlap between the unperturbed $53 \mathrm{~s}$ state and the trilobite state, as a function of the atom density $\rho$. The probability distribution is visualized through its mean and percentile curves and estimated from an ensemble as in Fig. 2

Energy shift and mixing with the s-state.-Comparing the behavior of high- $l$ states and s-states in a high density uncovers an important and experimentally relevant detail of the highdensity trilobites. Figure 3 shows the energy of the $n=50$ trilobite state and the energetically neighboring $53 \mathrm{~s}$ state as a function of $\rho$. Interaction with the perturbing atoms shifts both states to lower energies and, as predicted by Fermi [19], the s-state energy shift depends linearly on $\rho[15]$. However, as already seen in the previous results, the quasidegenerate manifold of high- $l$ states has much more freedom to optimize the electron density around variations in the local density. As a result the trilobite state shifts down in energy faster than the s-state as $\rho$ is increased.

As seen in Fig. 3 the $n=50$ trilobite crosses the 53 s state near $\rho=2 \times 10^{16} \mathrm{~cm}^{-3}$, creating hybrid states that contain some s-character. This is shown in Fig. 4 in more detail. Mixing with non-polar s-states also causes a dip in the dipole moment at this density in Fig. 2. As mentioned, mixing s-character into the trilobites is a highly sought-for property [9.-11] because without low- $l$ character the photoassociation of trilobite molecules is hindered by selection rules. In previous works it has been achieved by choosing a system with special resonances, but here it arises as a natural consequence of the variational flexibility of the high- $l$ states.

Outlook.-An atom density of $\rho \approx 10^{16} \mathrm{~cm}^{-3}$ at an ultracold temperature is, to our knowledge, approximately an order of magnitude higher than what is realized in current experiments. With higher $n$ the local electron wavelength at large $R$ and the available volume are larger, and thus the clustering regime is reached at lower densities [26]. On the other hand, $n \approx 50$ has the advantage that the clustering regime and the s-mixing regime occur at similar densities. As a high $\rho$ is only needed to make random clusters more probable in a homogeneous density, one possible option is to specifically increase the local density at some points.

To summarize, we have demonstrated that highly polar trilobite states persist and even thrive in a dense gas. This counterintuitive result emerges from a combination of perturbationinduced quantum scarring and randomly occurring atom clusters in the gas. Moreover, at a certain $n$-dependent density of the gas the states gain some s-character and thus become accessible with conventional photoassociation, overcoming a common obstacle with conventional dimer trilobites.

We thank B. Drury for useful discussions.

* perttu.luukko@iki.fi; Current address: Tampere University of Technology, Laboratory of Physics, Tampere, Finland

[1] C. H. Greene, A. S. Dickinson, and H. R. Sadeghpour, Phys. Rev. Lett. 85, 2458 (2000)

[2] C. H. Greene, E. L. Hamilton, H. Crowell, C. Vadla, and K. Niemax, Phys. Rev. Lett. 97, 233002 (2006)

[3] V. Bendkowsky, B. Butscher, J. Nipper, J. P. Shaffer, R. Löw, and T. Pfau, Nature 458, 1005 (2009)

[4] W. Li, T. Pohl, J. M. Rost, S. T. Rittenhouse, H. R. Sadeghpour, J. Nipper, B. Butscher, J. B. Balewski, V. Bendkowsky, R. Löw, and T. Pfau, Science 334, 1110 (2011)

[5] J. Tallant, S. T. Rittenhouse, D. Booth, H. R. Sadeghpour, and J. P. Shaffer, Phys. Rev. Lett. 109, 173202 (2012)

[6] M. A. Bellos, R. Carollo, J. Banerjee, E. E. Eyler, P. L. Gould, and W. C. Stwalley, Phys. Rev. Lett. 111, 053001 (2013).

[7] A. T. Krupp, A. Gaj, J. B. Balewski, P. Ilzhöfer, S. Hofferberth, R. Löw, T. Pfau, M. Kurz, and P. Schmelcher, Phys. Rev. Lett 112, $143008(2014)$

[8] D. A. Anderson, S. A. Miller, and G. Raithel, Phys. Rev. Lett. 112, 163201 (2014).

[9] D. Booth, S. T. Rittenhouse, J. Yang, H. R. Sadeghpour, and J. P. Shaffer, Science 348, 99 (2015)

[10] K. S. Kleinbach, F. Meinert, F. Engel, W. J. Kwon, R. Löw, T. Pfau, and G. Raithel, Phys. Rev. Lett. 118, 223001 (2017)

[11] M. T. Eiles and C. H. Greene, Phys. Rev. Lett. 115, 193201 (2015)

[12] V. Bendkowsky, B. Butscher, J. Nipper, J. B. Balewski, J. P. 
Shaffer, R. Löw, T. Pfau, W. Li, J. Stanojevic, T. Pohl, and J. M. Rost, Phys. Rev. Lett. 105, 163201 (2010)

[13] A. Gaj, A. T. Krupp, J. B. Balewski, R. Löw, S. Hofferberth, and T. Pfau, Nat. Commun. 5, 4546 (2014)

[14] M. Schlagmüller, T. C. Liebisch, F. Engel, K. S. Kleinbach, F. Böttcher, U. Hermann, K. M. Westphal, A. Gaj, R. Löw, S. Hofferberth, T. Pfau, J. Pérez-Ríos, and C. H. Greene, Phys. Rev. X 6, 031020 (2016)

[15] T. C. Liebisch, M. Schlagmüller, F. Engel, H. Nguyen, J. Balewski, G. Lochead, F. Böttcher, K. M. Westphal, K. S. Kleinbach, T. Schmid, A. Gaj, R. Löw, S. Hofferberth, T. Pfau, J. Pérez-Ríos, and C. H. Greene, J. Phys. B 49, 182001 (2016)

[16] I. C. H. Liu and J. M. Rost, Eur. Phys. J. D 40, 65 (2006)

[17] M. T. Eiles, J. Pérez-Ríos, F. Robicheaux, and C. H. Greene, J. Phys. B 49, 114005 (2016)

[18] P. J. J. Luukko, B. Drury, A. Klales, L. Kaplan, E. J. Heller, and E. Räsänen, Sci. Rep. 6, 37656 (2016)

[19] E. Fermi, Nuovo Cimento 11, 157 (1934).

[20] A. Omont, J. Phys. France 38, 1343 (1977)

[21] E. L. Hamilton, C. H. Greene, and H. R. Sadeghpour, J. Phys. B 35, L199 (2002)

[22] T. F. O’Malley, L. Spruch, and L. Rosenberg, J. Math. Phys. 2, 491 (1961)

[23] C. Bahrim, U. Thumm, and I. I. Fabrikant, J. Phys. B 34, L195 (2001)

[24] A. A. Khuskivadze, M. I. Chibisov, and I. I. Fabrikant, Phys. Rev. A 66, 042709 (2002)

[25] P. Ramachandran and G. Varoquaux, Comput. Sci. Eng. 13, 40 (2011)

[26] See Supplemental Material at [URL will be inserted by publisher] for a video of the trilobite state shown in Fig. 1 and for results with other principal quantum numbers $n$.

[27] E. J. Heller, Phys. Rev. Lett. 53, 1515 (1984).

[28] L. Kaplan, Nonlinearity 12, R1 (1999)

[29] The Bohr-Sommerfeld formula happens to be exact for hydrogen, making the resonant sets perfectly degenerate in the absence of quantum defects.

[30] J.-C. Gay, D. Delande, and A. Bommier, Phys. Rev. A 39, 6587 (1989)

[31] B. E. Granger, E. L. Hamilton, and C. H. Greene, Phys. Rev. A 64, 042508 (2001).

[32] M. Gardner, Sci. Am. 196, 166 (1957) 


\section{Polyatomic trilobite Rydberg molecules in a dense random gas}

\section{Supplementary material}

Other principal quantum numbers. - The main article describes trilobite states forming from the quasidegenerate manifold of states with principal quantum number $n=50$. For completeness, Figures 5,10 show the probability distributions of the trilobite state energy and dipole moment for other values of $n$. All cases show a similar qualitative behavior: highly polar trilobite states start to localize on clusters at some sufficiently high atom density, and at some density the states cross and mix with a s-state. For larger $n$, larger clusters can fit inside a single half-wavelength of the electron, and consequently highly polar trilobite states appear at lower atom densities. The calculations for $n=70$ and $n=90$ use a smaller ensemble of 1000 snapshots of $\left\{\boldsymbol{R}_{p}\right\}$.
For higher $n$ the cluster-induced trilobite states recover a smaller fraction of the dipole moment of the dimer case $(\langle P\rangle \approx 1)$. This is because as $n$ increases, the global minimum of the dimer Born-Oppenheimer potential occurs at a smaller fraction of $r_{\mathrm{c}}=2 n^{2}$. The radii near this minimum is where the cluster can dominate over other perturbing atoms. It is important to note that these radii are also where stable dimer molecules are found, i.e., the cluster-induced trilobite states have similar dipole moments as the dimer trilobites in a stable configuration, even at higher $n$.

Importance of clusters. - As discussed in the main article, in a high atom density the trilobite states localize on randomly occurring clusters of atoms. Figure 11 illustrates the importance of clusters by showing the probability distribution of the trilobite state dipole moment in a calculation where most clusters are excluded by enforcing a minimal distance between atoms. Without clusters there is no increase in dipole moment at high densities. 


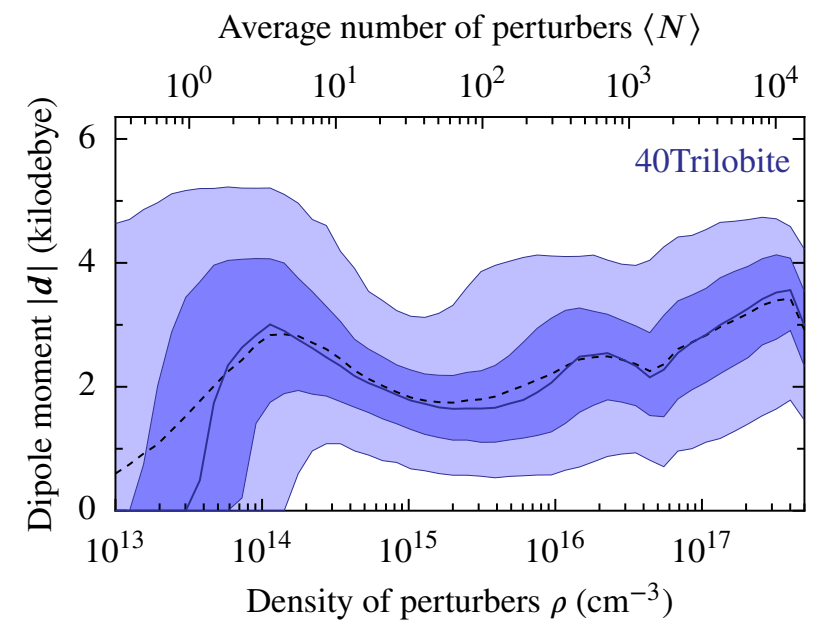

FIG. 5. Probability distribution of the dipole moment, as Fig. 2 but with $n=40$.

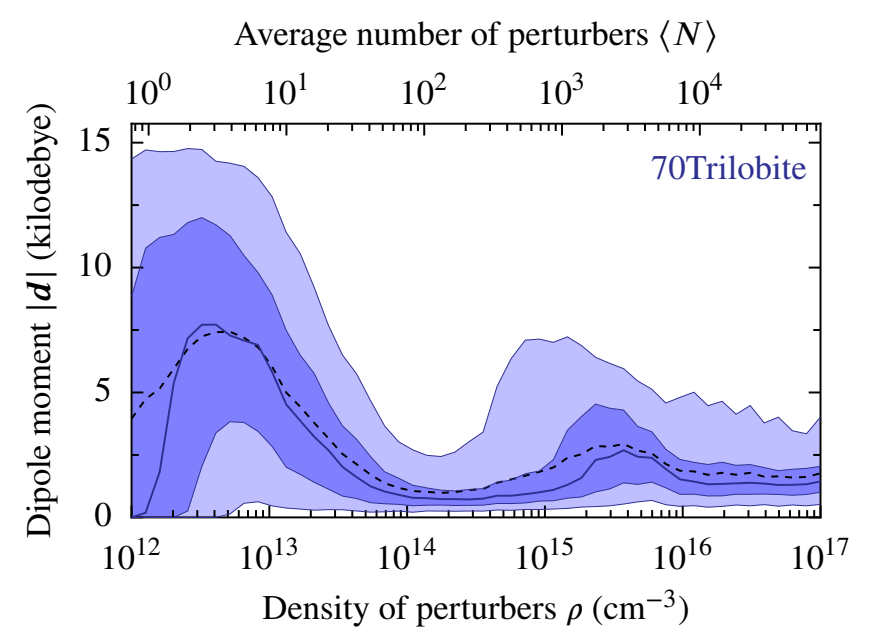

FIG. 7. Probability distribution of the dipole moment, as Fig. 2 but with $n=70$.

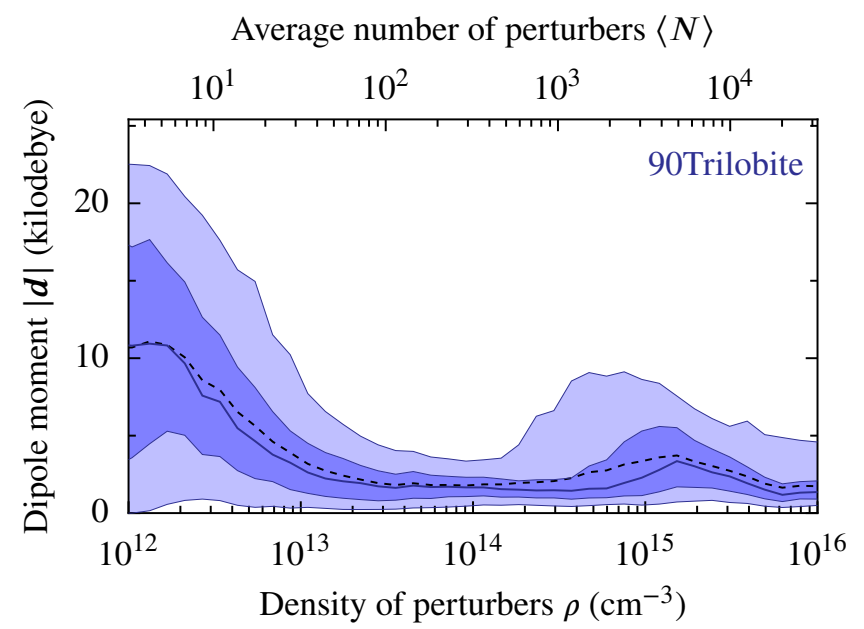

FIG. 9. Probability distribution of the dipole moment, as Fig. 2 but with $n=90$.

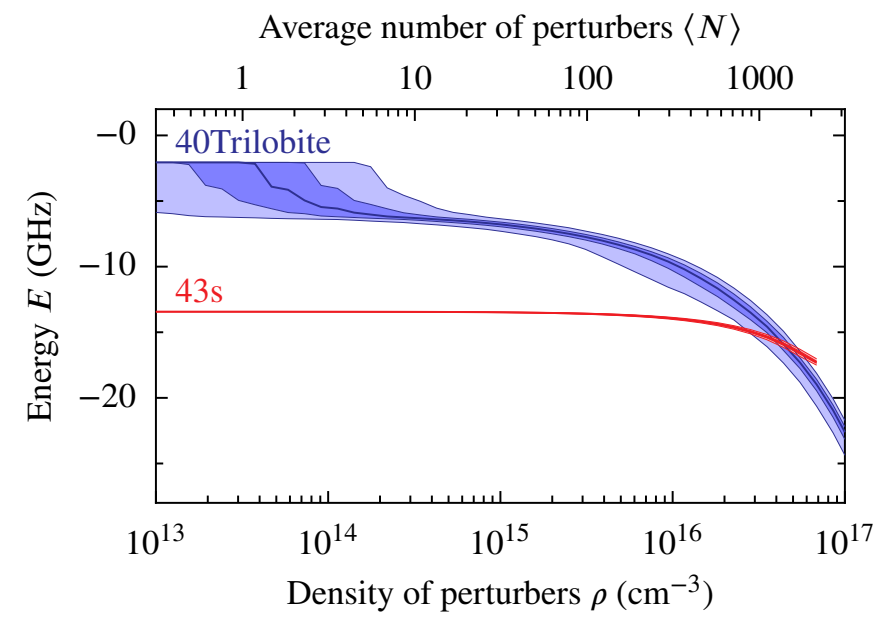

FIG. 6. Probability distribution of the energy, as Fig. 3 but with $n=40$.

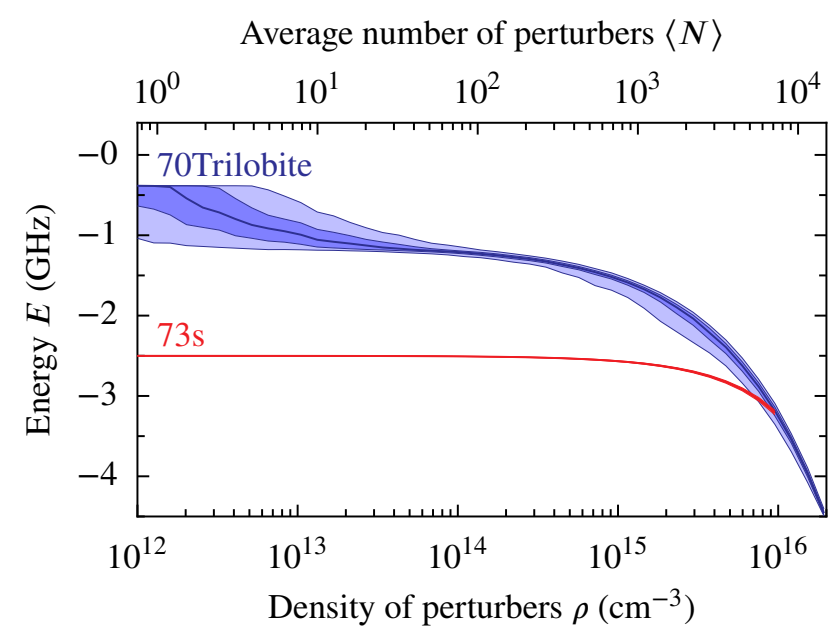

FIG. 8. Probability distribution of the energy, as Fig. 3 but with $n=70$.

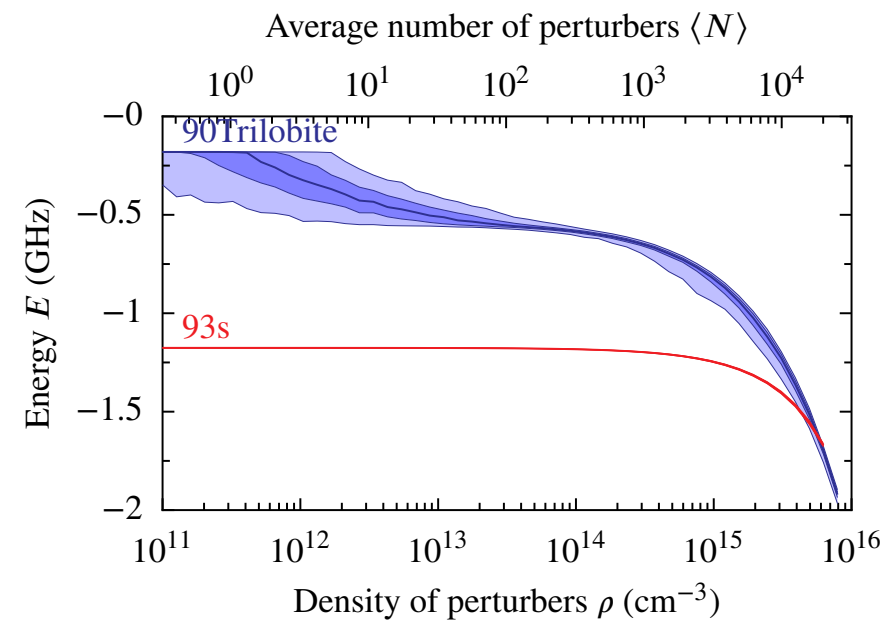

FIG. 10. Probability distribution of the energy, as Fig. 3 but with $n=90$. 
Average number of perturbers $\langle N\rangle$

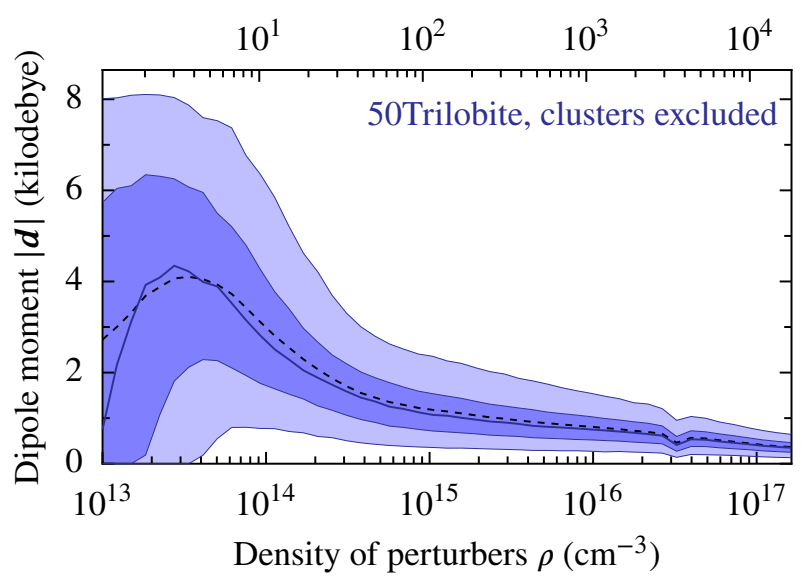

FIG. 11. Probability distribution of the dipole moment, as Fig. 2 but excluding atom clustering by enforcing a minimal distance of 300 a.u. between atoms. 